\title{
Daylength and Twilight variations in Svalbard during the month of October
}

\section{ANALYSIS PAPER}

Pardis Tabaee Damavandi

\section{INTRODUCTION}

Longyearbyen is the capital of the northern arctic Norwegian archipelago inhabited by exceptional wildlife, including Greenland right whales, Walruses, Polar Bears, Arctic foxes, ringed seals, ptarmigans, arctic hares and by 2650 people approximately.

In this part of the world, lifestyle differs, mainly due to it exhibiting Polar nights in the AutumnWinter months and the Midnight Sun in the Summer.

These phenomena as well as the Aurora Borealis, are extremely charming, however, they may pose a risk to vitamin $D$ deficiency to both the animal and human populations due to the lack of direct sun exposure; they too, may affect circadian rhythms, however, ultraviolet UVB radiation, which is required for the conversion of Provitamin $D$ into Vitamin $D$, has been measured in the presence of Civil twilight, which is a type of twilight characterised by a yellow sky mixed with azure shades, compensating for the missing UVB in "daylight-absent months". It is also imperative that in those regions pollution stays at a minimum to favour a maximal radiation absorption, which although is less than direct daylight exposure, it impacts the quality of the radiation by not "trapping" nor filtering it.

This analysis paper demonstrates how daylight times vary in the Polar region and particularly in the town of Longyearbyen, and how civil twilight compensates with squandered daylight during polar nights.

\section{TWILIGHT DEPICTIONS}

Twilight is defined as the period between the sunset, or sunrise, until dusk. There are four known forms of twilight, civil twilight, which has already been described, followed by nautical twilight, where the sky turns vermilion with blue splashes, and lastly, astronomical twilight, where a crimson sky fades and blends into a prevalently dark navy blue atmosphere ${ }^{2}$.

During twilight hours, the sun cannot, due to its geolocation, elevate beyond the horizon. Below, three scenarios are analysed. 
a) In Longyearbyen, Svalbard, Norway. (Figure 1) The charts in figure 1 show that daylight decreases polynomially and almost linearly whilst civil twilight remains constant and a slight increase the last 7 days of October (after time change). The nautical twilight slightly increase, but remains otherwise constant, whereas astronomical twilight and night drop around the $8^{\text {th }}$ day of the month, and then significantly increase, overtaking daylight and the lighter, "UVB filled", twilight hours, which only occupy $40 \%$ of the days in the beginning of the month and reduce to $30 \%$ by the end of the month. More specifically, daylight hours vanish from 10 hours on $1^{\text {st }}$ October, 2020, and civil twilight increases by 7 hours by the end of the month, compensating for the lack of daylight.

\section{Longyearbyen (NO), Arctic}

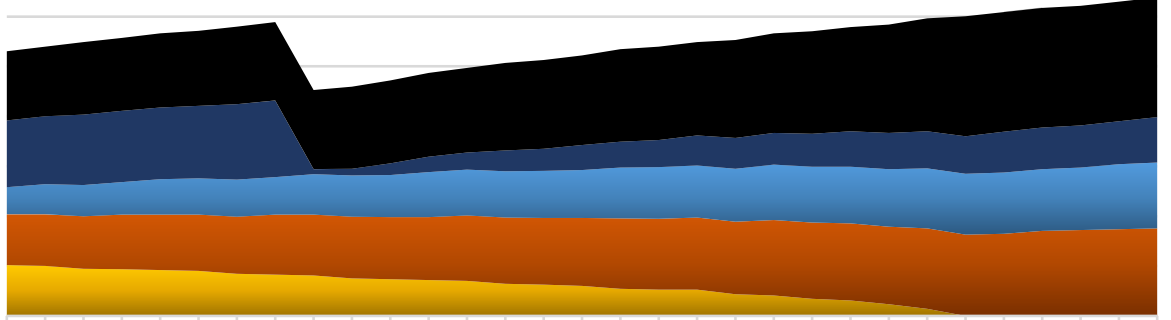

$142 \quad 3 \quad 4 \quad 5 \quad 6 \quad 7 \quad 8 \quad 910111213141516171819202122232425262728293031$

Length Day

- Length Nautical Twilight

- Length Night
- Length Civil Twilight

- Length Astronomical twilight

\section{Figure 1.}

a) Variations of daylight, twilight and night hours in the month of October 2020.

\section{Longyearbyen (NO), Arctic}

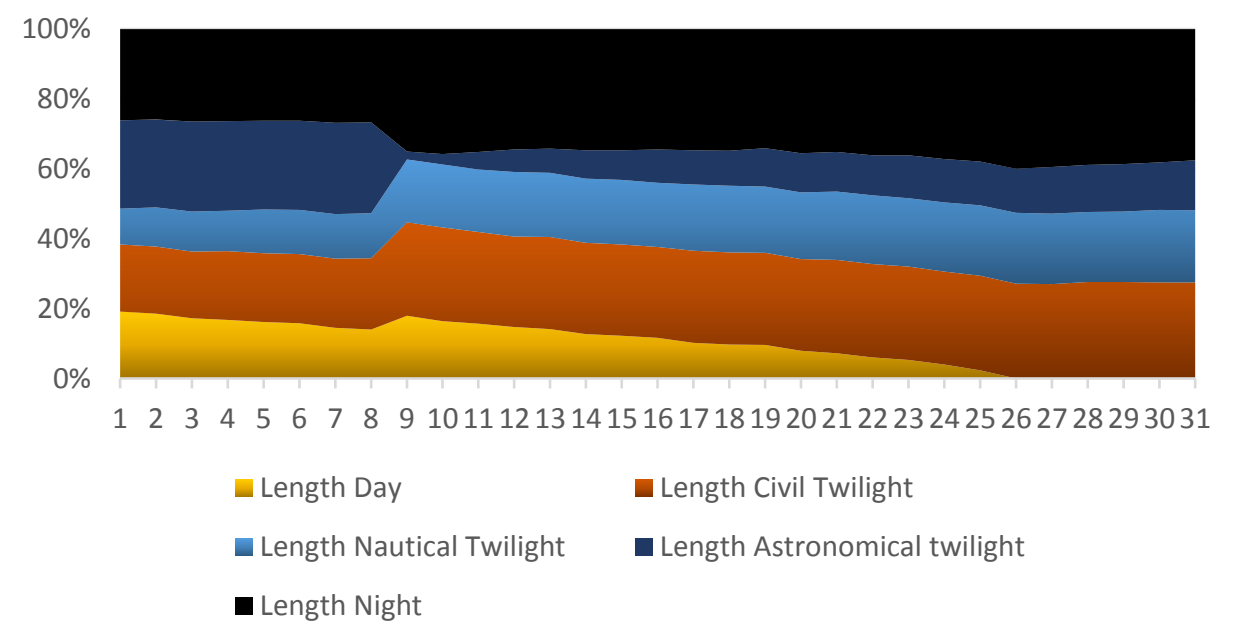


b) Similar to $a$ ) but with percentages. Dark hours overcome the daylight and civil twilight hours, reducing UVB exposure.

b) In Europe and the US. (Figure 2) The charts in figure 2 depict daylight, twilight and night hours in temperate regions, which seems to be more constant, due to the geological location, with slight decrements of daylight hours. 

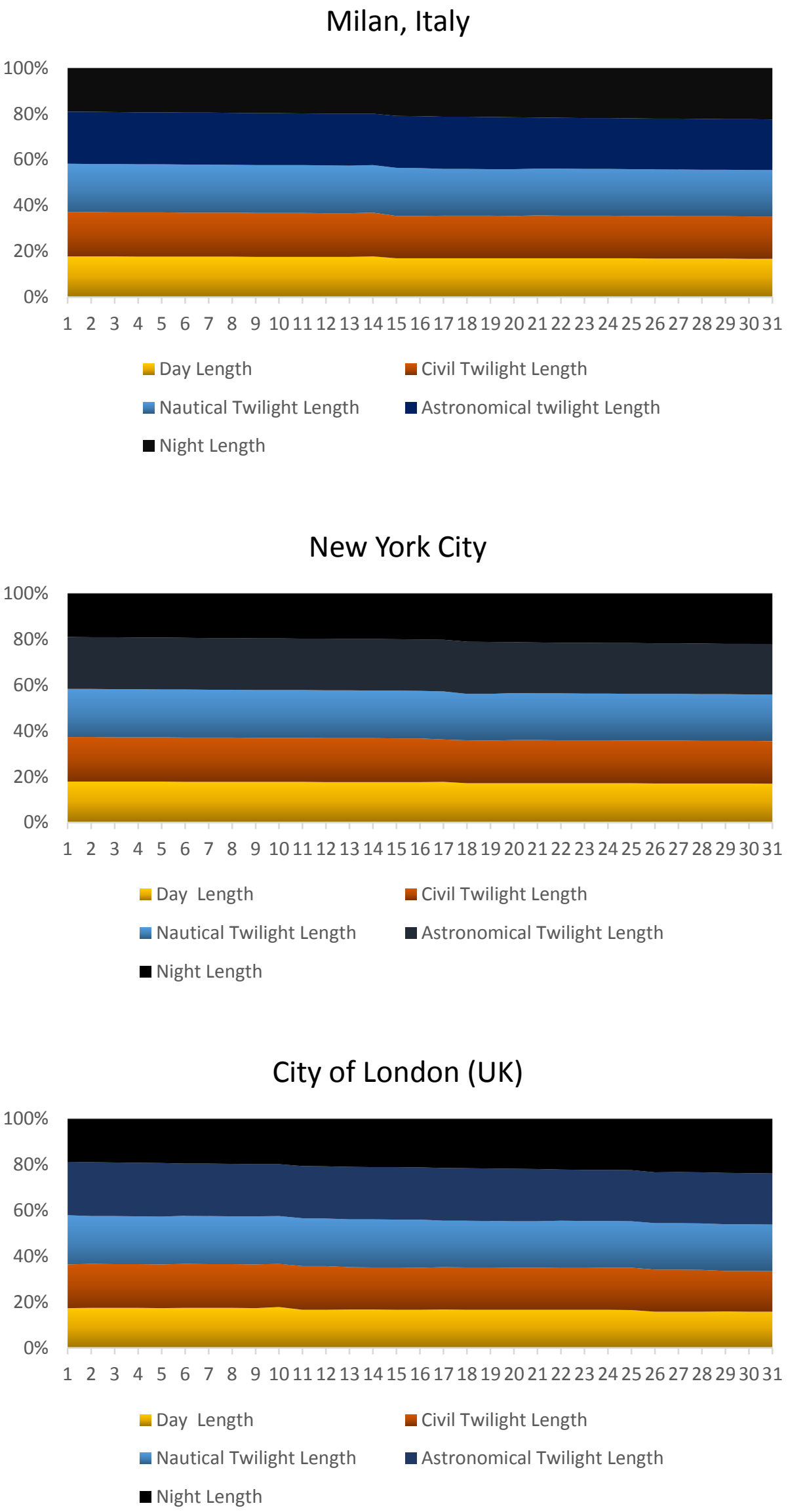

Figure 2. As it can be viewed the daylight $(\mathrm{h})$ is more or less the same in cities of temperate areas. 
c) Differences between temperate and polar regions (Figure 3). The chart proves that the decrease in daylight in the arctic region is far more drastic than the moderatelight decrease observed in the Temperate areas.

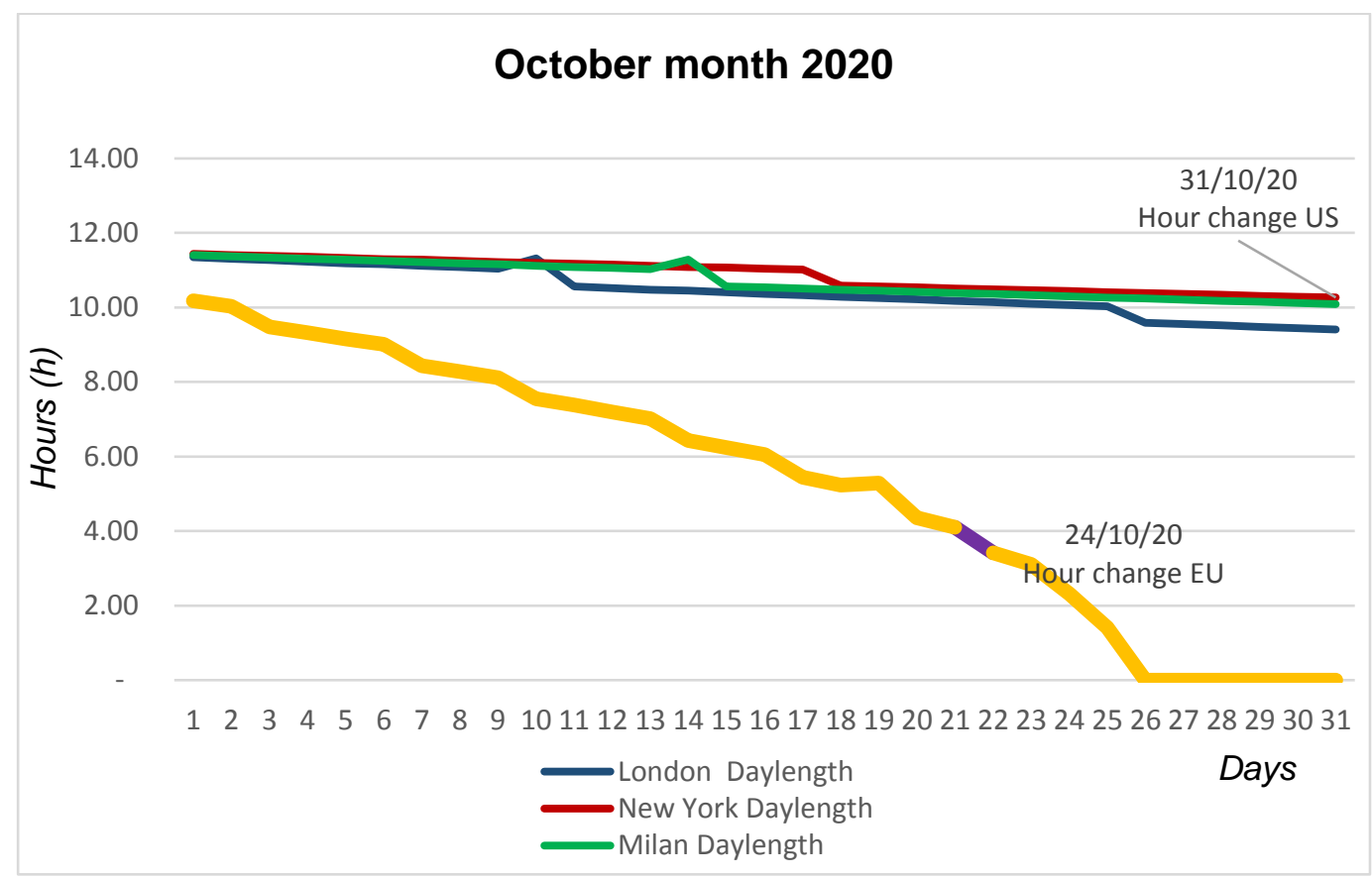

Figure 3. The chart determines an initial decrease of half a hour daylight for all cities in the temperate regions with a further half hour reduction in London with the hour change, but the reduction in light exposure is very constant, compared to the 15-minute to 1 hour daily decrements observed in the Arctic region.

We could say that the amount of daylight for the yellow polynomial curve for the month of October:

$y=7 \cdot 10^{-6} x^{5}-0.0005 x^{4}+0.0112 x^{3}-0.1134 x^{2}+0.1759 x+9.9836$

since $x^{5}, x^{4}, x^{3}$ and $x^{2} \rightarrow 0$ (it can be approximated to)

$\mathrm{y}=-0.1759 \mathrm{x}+9.9836$

(depicting Longyearbyen daylight reduction), with a negative slope denoting a decrement,

is quantified by the area under the curve

$$
\int_{f d o}^{l d o}-0.1759 \mathrm{x} 2+9.9836 \mathrm{x}+C
$$

where $I d O$ is the last day of October daylight, and fdO is the first day of October daylight.

\section{CONCLUSION}

Daylight lengths vary across the northern hemisphere and the difference in the daily and monthly decrements for the month of October 2020 between the temperate cities and the polar 
city have at this point been interpreted. The relevance is not just for vitamin D deficiency, as both temperate and arctic region populations require vitamin $D$ supplements from a reduced daylight exposure, but lifestyle fluctuates considerably in the polar region with midnight sun days and polar nights. Polar nights cover the arctic skies between the end of October until mid-February months, with twilight hours lasting longer and "unusually" for temperate city inhabitants. This has an impact on temperatures and light, but also on the circadian rhythms of both wildlife and human arctic populations. The amount of daylight reduction for the month of October c. y. was examined and calculated with an approximated definite integral. This integral simply analyses and quantifies the amount of daylight missing and it also shows the drastic decrement in the month of October.

\section{Declaration}

No conflicts of interest to disclose.

\section{References}

1. Spitschan, M., Aguirre, G. K., Brainard, D. H., \& Sweeney, A. M. (2016). Variation of outdoor illumination as a function of solar elevation and light pollution. Scientific reports, 6 , 26756. https://doi.org/10.1038/srep26756

2. Brastad, I., 2020. Sunrise And Sunset Times In Longyearbyen, October 2020. [online] Timeanddate.com.

https://www.timeanddate.com/sun/norway/longyearbyen?month=10\&year=2020 [Accessed 10 November 2020].

(C) 2020 by Pardis Tabaee Damavandi. All rights reserved. No part of this publication may be reproduced, distributed, or transmitted in any form or by any means, including photocopying, recording, or other electronic or mechanical methods, without the prior written permission of the publisher, except in the case of brief quotations embodied in critical reviews and certain other non-commercial uses permitted by copyright law. 